\title{
Disparidad de criterios respecto del hecho gravado y contribuyente en el impuesto específico a la actividad minera (Corte de Apelaciones de Rancagua; Corte Suprema)
}

\author{
Comentario de Patricio Masbernat Muñoz ${ }^{1}$
}

Rancagua, veinticinco de enero de dos mil once.

\section{VISTOS Y CONSIDERANDO:}

1.- Que en estos autos Rol No10.005-2008, seguidos ante el Director Regional del Servicio de Impuestos Internos, en virtud del procedimiento de reclamación establecido en los artículos 123 y siguientes del Código Tributario, y que fuera impetrado por Minera Valle Central S.A. contra los giros deducidos a su respecto, $\mathbf{N}^{\mathrm{os}} 101093538-1$; 101093548-9; 101093558-6; 101093578-0; 101093598-5; 101093618-3; 101093658-2; 101093678-7; 101093688-4; 101093718-K; 101093748-1; y 101093758-9, todos de fecha 11 de Diciembre de 2007, se ha deducido recurso de apelación por la reclamante en contra de la sentencia dictada con fecha 20 de Julio de 2010, que niega lugar a la reclamación deducida, confirmando los giros objeto de la misma.

2.- Que dichos giros contienen la determinación de reajustes, intereses y multas respecto de Pagos Provisionales Mensuales con obligación anual cumplida, que Minera Valle Central S.A. debió pagar, según plantea el Servicio de Impuestos Internos, en los períodos tributarios de Enero a Diciembre de 2006, los cuales no fueron enterados en arcas fiscales, de conformidad con lo dispuesto en el artículo 84 letra h) en relación con el artículo 64 bis, ambos de la Ley de Impuesto a la Renta, y que se refieren al impuesto específico a la actividad minera.

3.- Que Minera Valle Central S.A. funda su recurso de apelación en la circunstancia de no reunirse en su caso los supuestos normativos que configuran el hecho gravado por el impuesto referido, por cuanto no es titular ni explota pertenencias mineras, ni extrae, por lo mismo, sustancias minerales de carácter concesible.

Señala al respecto que el fallo del Sr. Director Regional se funda únicamente en el Oficio $\mathrm{N}^{\circ} 1.164$ de fecha 28 de Mayo de 2007, del Servicio de Impuestos Internos (acompañado a fojas 133), el cual, consultado sobre el alcance del artículo 64 bis de la

${ }^{1}$ El presente trabajo se enmarca en el Proyecto FONDECYT N ${ }^{\circ} 11100190$, "Construcción de una dogmática de los principios materiales de la tributación en Chile, a la luz del Derecho Comparado”, ejecución año 2011/2012, del cual el autor es investigador responsable. Este trabajo está dedicado a Lucas. 
Ley de Impuesto a la Renta, expresa que es plenamente aplicable al reclamante, respuesta que este último rechaza por estimarla contraria a Derecho, haciendo hincapié en que tal informe no puede ser obligatorio para los Tribunales de Justicia ni para los contribuyentes.

4.- Que en cuanto al fondo, la reclamante sostiene latamente en su escrito de apelación de fojas 182 que no es ni puede ser sujeto del impuesto específico a la actividad minera, toda vez que este se aplica, de acuerdo al artículo 64 bis ya citado, sobre las rentas operacionales de la actividad minera obtenidas por un explotador minero, calidad que no ostenta, puesto que no es titular de una concesión de explotación ni realiza, por lo tanto, extracción alguna de sustancias minerales concesibles, en los términos del artículo 116 del Código de Minería. En tal sentido, señala que su actividad principal es la recuperación, a través de procesos físicos y químicos, del cobre y molibdeno contenidos en el relave generado por la planta de beneficio de minerales de la División El Teniente de la Corporación Nacional del Cobre de Chile, lo que hace en virtud de un contrato firmado con dicha empresa con fecha 9 de Septiembre de 1991 (cuya copia aparece acompañada a fojas 15 de autos), consistente en la venta de derechos de procesamiento de los relaves frescos de División El Teniente, con el objeto que la compradora extraiga los minerales antes mencionados, los haga suyos, restituyendo el resto a la vendedora. De este modo, explica, no se hace dueño de dichas sustancias minerales mediante la extracción ni a través de la aprehensión, en los términos utilizados por el artículo 116, incisos segundo y tercero del código del ramo, ni menos puede aplicársele lo dispuesto en el artículo $6^{\circ}$ de dicho texto legal, relativo a las sustancias concesibles que los desmontes, relaves o escorias contengan, puesto que solo podría obtener, en este caso, concesión sobre éstos en la medida que la pertenencia o el establecimiento de beneficio de que proceden estuvieren extinguidos o abandonados, lo que no sucede en el caso de autos, toda vez que se trata, como ya se dijo, de relaves de Codelco, y por lo tanto de sustancias minerales de las que este se ha hecho dueño, en primer término, por haberlas extraído.

Expresa además que si se desconociera el dominio de Codelco sobre tales productos, estaríamos en presencia de una venta de cosa ajena, puesto que los minerales extraídos continuarían en poder del Estado.

En fin, señala por último que la venta que la citada empresa ha hecho a Minera Valle Central S.A. ya ha sido gravada por el impuesto específico aludido, y por lo tanto las ventas que a su vez haga la reclamante no pueden ser gravadas nuevamente, puesto que no estamos en presencia de un impuesto plurifásico.

Solicita, en consecuencia, se enmiende conforme a derecho la sentencia apelada, revocándola en todas sus partes y resolviéndose en su lugar que se da lugar y se acoge el reclamo por ella interpuesto en contra de los giros antes singularizados, dejándolos sin efecto en su totalidad.

5.- Que, a la luz de lo expuesto por la apelante, se trata aquí de dilucidar un punto de Derecho, cual es determinar si, de acuerdo a lo dispuesto por el artículo 64 bis de Código de Minería, puede ser la reclamante considerada sujeto del impuesto específico a la actividad minera.

6.- Que al respecto, cabe señalar en primer término que la norma en alusión parte diciendo lo siguiente: "Establécese un impuesto específico a la renta operacional de la 
actividad minera obtenida por un explotador minero". Luego define "explotador minero" como "toda persona natural o jurídica que extraiga sustancias minerales de carácter concesible y las venda en cualquier estado productivo en que se encuentren".

Ahora bien, huelga decir que, al tenor de lo dispuesto en el artículo $1^{\circ}$ del Código de Minería y del artículo 19 N 24 de la Constitución Política de la República, teniendo el Estado el dominio absoluto, exclusivo, inalienable e imprescriptible de todas las minas, la única vía que nuestra legislación establece para que otros se hagan dueños de manera originaria, vale decir, mediante su extracción directa, de las sustancias minerales que de ellas provienen, es la respectiva concesión de explotación, lo que nos remite directamente al Título VIII del citado código, "De los Derechos y Obligaciones de los Concesionarios Mineros", que parte por señalar, en su artículo 107, que "solo desde que quede constituida la concesión, el titular podrá efectuar los trabajos que estime necesarios para la exploración y, en su caso, también para la explotación de la mina, según la especie de concesión de que se trate". Es preciso tener en consideración en este punto que el reclamante ha señalado que no es titular de pertenencias mineras, circunstancia que el Servicio de Impuestos Internos no ha rebatido.

7.- Que, si bien en este punto el sentenciador ha aseverado, fundándose para ello en el Oficio $\mathrm{N}^{\circ} 1164$ antes mencionado, que la reclamante es una persona jurídica que se dedica a la extracción de cobre y molibdeno para su posterior venta, y por lo tanto califica dentro del concepto de "explotador minero" en los términos del artículo 64 bis de la Ley de Impuesto a la Renta, disposición que no exigiría, según sostiene, que se trate de la extracción y venta de sustancias que hayan sido objeto de una concesión, sino que tengan la aptitud legal para ello, no es correcto, a nuestro juicio, su razonar, toda vez que, si bien el cobre y el molibdeno son substancias concesibles por su naturaleza, no lo son, en este caso concreto, desde el punto de vista jurídico, toda vez que, de acuerdo al artículo $6^{\circ}$, inciso segundo del Código de Minería, solo puede constituirse concesión sobre las sustancias minerales concesibles que los relaves contengan, cuando el establecimiento de beneficio del cual provienen se encontrare abandonado, situación que, ciertamente, no se da en la hipótesis de autos.

8.- Que siguiendo con la norma en análisis, cabe observar que, antes de entrar al caso excepcional comentado en el numeral anterior, dicha disposición parte por fijar, en su inciso primero, una regla general en los siguientes términos: "los desmontes son cosas accesorias de la pertenencia de que proceden, y los relaves y escorias lo son del establecimiento de beneficio de que provienen". Pues bien, relacionado esto con lo dispuesto en el artículo 116, incisos segundo y tercero, resulta obvio considerar que, siendo el concesionario quien tiene el derecho de hacerse dueño de todas las sustancias minerales concesibles que extraiga dentro de los límites de su pertenencia, debe entenderse que en el caso de autos es Codelco quien en primer lugar ha adquirido el dominio de los minerales contenidos en el relave, porque ha sido este quien los ha separado del depósito natural del que formaban parte, independiente que los haya transferido a un tercero con anterioridad a su beneficio.

Dicha compraventa no es más que la expresión de su facultad de vender el producto minero del que se ha hecho dueño, cualquiera sea el estado productivo en que se encuentre, 
máxime si en esa venta se precisó que el objeto de la venta es el cobre y molibdeno que el comprador pueda extraer del relave que accede a su establecimiento de beneficio.

9.- Que, de este modo, retornamos a lo dispuesto por el artículo 64 bis de la Ley de Impuesto a la Renta tantas veces citado, para observar que, luego de definir al sujeto del impuesto, señala que debe entenderse por "producto minero, la sustancia mineral de carácter concesible ya extraída haya o no sido objeto de beneficio, en cualquier estado productivo en que se encuentre", para luego definir "venta", vale decir, la actividad generadora de la renta imponible, como "todo acto jurídico celebrado por el explotador minero que tenga por finalidad o pueda producir el efecto de transferir la propiedad de un producto minero".

De lo anterior se desprende que la aseveración hecha por Minera Valle Central S.A., en orden a sostener que no es ni puede ser calificada como "explotador minero" (y por lo tanto, sujeto del impuesto), es acertada, toda vez que no es titular de concesión alguna, ni menos pudo haberse hecho dueña de los minerales con los cuales comercia por "aprehensión", como lo ha pretendido el sentenciador, dada la limitación que al respecto establece el artículo $6^{\circ}$ del código en estudio, se torna lógica y comprensible, toda vez que, existiendo un contrato de compraventa con Codelco Chile respecto del cobre y molibdeno existentes en sus relaves, no cabe más que suponer que ha adquirido tales sustancias minerales por compraventa, y no por explotación directa que, como ya vimos, en su situación es legalmente imposible.

10.- Que, en fin, de lo expuesto resulta que el sujeto del impuesto específico a la actividad minera no podría ser otro que el vendedor, y por lo tanto, pretender gravar también a la reclamante significa en efecto aplicar dos veces el mismo tributo a idéntico objeto, lo que no se condice con la naturaleza del impuesto en análisis, el que, como bien ha dicho la apelante, no puede ser considerado plurifásico.

Por todo lo expuesto y vistos, además, lo dispuesto en los artículos 186 y siguientes del Código de Procedimiento Civil; artículo 139, 141 y siguientes del Código Tributario; artículo 64 bis de la Ley de Impuesto a la Renta y artículos $6^{\circ}$ y 116 del Código de Minería, se declara que se acoge el recurso de apelación deducido por la reclamante Minera Valle Central S.A. en el sentido que se revoca la sentencia apelada de fecha 20 de Julio de 2010, rolante a fojas 175 de autos, contenida en Resolución $\mathrm{N}^{\circ} 39$ del Tribunal Tributario de la VI Dirección Regional Rancagua del Servicio de Impuestos Internos, en cuanto rechaza la reclamación formulada por la apelante, y se resuelve en cambio que ha lugar en todas sus partes al reclamo interpuesto por Minera Valle Central S.A. en contra del Servicio de Impuestos Internos, respecto de los giros $\mathbf{N}^{\text {os }} 101093538-1$; 101093548-9; 101093558-6; 101093578-0; 101093598-5; 101093618-3; 1010936582; 101093678-7; 101093688-4; 101093718-K; 101093748-1; y 101093758-9, todos de fecha 11 de Diciembre de 2007, los que se dejan sin efecto en su totalidad.

Acordada con el voto en contra del Ministro Sr. Aránguiz quien en cambio, estuvo por confirmar la sentencia en alzada de acuerdo a sus propios fundamentos”.

Corte de Apelaciones De Rancagua - Primera Sala - 25.01.2011 - Minera Valle Central S.A. C/SII - Rol 690-2010 - Ministros Srs. Carlos Aránguiz Zúñiga, Raúl Mera Muñoz, Abogada Integrante Sra. Francisca Alvarez Hurtado. 
"Santiago, veintiocho de noviembre de dos mil doce

\section{VISTOS:}

En estos autos Rol No 2.518-2011 de esta Corte Suprema, sobre procedimiento de reclamación tributaria, iniciado por el contribuyente Minera Valle Central S.A., se dictó sentencia de primer grado el 20 de julio de 2010, la que rola a fojas 175 y siguientes, por la que se desestimó el reclamo de autos. La anterior decisión fue recurrida de apelación por la reclamante mediante presentación que rola a fojas 182 , la que fue resuelta por la Corte de Apelaciones de Rancagua el 25 de enero de 2011 y que rola a fojas 235 y siguientes, en virtud de la cual revocó la de primer grado, y en su lugar declaró, que la referida sociedad no se encuentra afecta al Impuesto Específico a la Actividad Minera descrito en el artículo 64 bis de la Ley de Impuesto a la Renta, dejando sin efecto los giros cursados bajo ese presupuesto.

A fojas 241 y siguientes, la defensa del Fisco dedujo recurso de casación en el fondo en contra de la sentencia anterior.

A fojas 262, se trajeron los autos en relación.

\section{CONSIDERANDO:}

Primero: Que en el recurso se señala como antecedente previo que el Servicio de Impuestos Internos emitió doce giros, todos de 11 de diciembre de 2007, basados en estimar que Minera Valle Central S.A., dadas las actividades desarrolladas al amparo de un contrato celebrado con Codelco Chile, División El Teniente, consistentes en extraer cobre y molibdeno desde relaves de la anterior, se encuentra afecta al pago del Impuesto Específico a la Actividad Minera señalado en el artículo 64 bis del DL 824, no obstante lo cual, los sentenciadores de segundo grado, vulnerando los artículos 2 del Código Tributario, 20 y 1.560 del Código Civil, además del ya referido al impuesto mismo, acogieron erradamente el reclamo del contribuyente, dejando sin efecto los giros respectivos.

SEgundo: Que, la infracción de los artículos $2^{\circ}$ del Código Tributario y 64 bis de la Ley de Impuesto a la Renta, se produjo al desatenderse el texto expreso de la primera, que conmina solo en lo no previsto por dicho cuerpo legal a aplicar las normas el derecho común, de forma tal que encontrándose definida la materia en esa sede, no cabe remitirse a otros textos, lo que fue flagrantemente infringido por la Corte de Alzada, al fundar su fallo revocatorio en conceptos extraídos del Código de Minería, desconociendo la especialidad del artículo 64 bis de la Ley de Impuesto a la Renta, que describe el impuesto y define los conceptos de: "explotador minero", "producto minero" y "venta", los que fueron omitidos para aplicar un estatuto diferente y más restringido.

Tercero: Que, más adelante, se agrega que para la Ley de Impuesto a la Renta, las exigencias para ser objeto del impuesto son: a) que se trate de una persona natural o jurídica; b) que extraiga sustancias minerales de carácter concesible, y c) las venda e cualquier estado 
productivo que se encuentren, sin requerir la existencia de una concesión minera de explotación, destacando al efecto la expresión legal "de carácter concesible", caso contrario a lo que ocurre en el Código de Minería, donde es imprescindible la respectiva concesión de explotación de los relaves -que fue el que los juridiscentes de segundo grado privilegiaron-, lo que implicó establecer requisitos no previstos en la ley para la procedencia del impuesto.

Cunto: Que, en cuanto al artículo 20 del Código Civil, referido al sentido natural y obvio de la palabra "extracción", que no se encuentra definida legalmente, para lo cual cita la 22 $2^{\mathrm{a}}$ versión del Diccionario de la Real Academia Española, la define como "acción $y$ efecto de extraer". Y el verbo "extraer", en su tercera acepción, consiste en "Obtener uno de los componentes de un cuerpo por la acción de disolventes u otros medios", que es el concepto que debió seguir la Corte de Apelaciones de Rancagua, lo que debió llevarla a concluir que la contribuyente de autos realiza actividades extractivas de sustancias minerales concesibles, en este caso obtener cobre y molibdeno generados por los relaves de la División El Teniente de Codelco.

Finalmente, en cuanto al artículo 1.560 del Código Civil, su trasgresión se produjo al desconocer su texto, que da preponderancia a la intención de los contratantes, toda vez que lo celebrado entre la Minera de autos y Codelco fue la venta de derechos de procesamiento de relaves, esto es, el derecho a extraer los minerales contenidos en ellos, actividad de extracción que es llevada a cabo por Valle Central S.A., y la venta de cobre y molibdeno a terceros es un segundo contrato diferente del anterior, aspectos que fueron confundidos por el tribunal de segundo grado, al otorgarle efectos diversos a los celebrados por las partes, señalando que el primero se refiere a la venta de esos minerales, lo que por lo expresado, no es efectivo.

Quinto: Que, por último, precisa que las anteriores infracciones tuvieron influencia sustancial en lo dispositivo del fallo, toda vez que de haber aplicado exclusivamente las normas especiales contenidas en la Ley de Impuesto a la Renta y los efectos del contrato entre las partes, necesariamente habrían llegado a la conclusión que la reclamante de autos sí ejerce actividades de extracción de minerales y se encuentra dentro del concepto de explotador minero otorgado por el Artículo 64 bis del DL 824, debiendo mantener los giros emitidos por el Servicio de Impuesto Internos, por todo lo cual solicita que se acoja su recurso, se anule la sentencia recurrida y en su reemplazo se proceda a dictar otra, por la que se confirme el fallo de primer grado, con costas.

Sexto: Que a fin de resolver acerca de las infracciones denunciadas en el libelo, es necesario consignar que los jueces del fondo, en lo pertinente, dieron por establecidos como hechos que no son discutidos por las partes del pleito, que la reclamante Sociedad Minera Valle Central S.A., en virtud de un contrato celebrado el 9 de septiembre de 1.991 con Codelco-Chile, recupera cobre y molibdeno desde los relaves frescos producidos por las plantas concentradoras de la División El Teniente de Codelco-Chile, minerales que posteriormente son vendidos a terceros. 
SÉPTimo: Que, fijada la anterior realidad, se puede advertir que la cuestión jurídica propuesta se reduce a dilucidar si las labores descritas precedentemente, se encuadran o no en los presupuestos que especifica el artículo 64 bis de la Ley de Impuesto a la Renta (D.L. 824), que hace aplicable el Impuesto Específico a la Actividad Minera, y de esa manera determinar sin son efectivos los yerros denunciados en el libelo de fojas 241 .

Octavo: Que, por la citada disposición se establece como hecho gravado del impuesto específico a la renta operacional, toda actividad minera obtenida por un explotador minero, el que se regula conforme a tasas que se aplican en relación a una tabla de valores equivalentes a ventas anuales en toneladas métricas de cobre fino, estableciendo como sujeto pasivo del mismo al explotador minero, que se define en la misma norma como toda persona natural o jurídica que extraiga sustancias minerales de carácter concesible y las venda en cualquier estado productivo e que se encuentren. A continuación se establece que el producto minero constituye la sustancia mineral de carácter concesible ya extraída, haya o no sido objeto de beneficio y en cualquier estado productivo que se encuentre; en tanto que por venta, se comprende todo acto jurídico celebrado por el explotador minero que tenga por finalidad o pueda producir el efecto de transferir la propiedad de un producto minero.

De lo anterior se infiere que la norma describe, precisa y define todos los elementos de su estructura impositiva, de manera tal que no cabía aplicar en la especie lo dispuesto en el artículo $2^{\circ}$ del Código Tributario, que permite, en lo no previsto en dicho texto o leyes tributarias, recurrir a las normas del derecho común contenidas en leyes generales o especiales, situación que desde ya permite vislumbrar como efectivo uno de los yerros de derecho que consigna la defensa fiscal en su recurso.

En efecto, tal como se alegó, los jueces de alzada, erradamente, dieron preferencia a las normas del Código de Minería, en particular las contenidas en su Título VIII "De los Derechos y Obligaciones de los Concesionarios Mineros”, en circunstancias que ello no era posible al estar ya regulado en la ley respectiva, los diferentes presupuestos del tributo en análisis en la que reciben un especial tratamiento.

Noveno: Que, en consecuencia, corresponde ahora contrastar la realidad fáctica descrita en el motivo sexto de la presente sentencia con lo expresado en el fundamento precedente, para determinar si la reclamante de autos cumple o no las exigencias para constituirse en sujeto pasivo del impuesto descrito en el artículo 64 bis de la Ley de Impuesto a la Renta.

En ese orden, Minera Valle Central es una persona jurídica constituida como sociedad anónima cerrada, cuyo giro es el tratamiento de concentrado de cobre y que como efecto del contrato celebrado con la División El Teniente de Codelco Chile el 9 de septiembre de 1.991, desarrolla labores de extracción y recuperación mediante procesos físicos y químicos de cobre y molibdeno contenidos en relaves frescos de la propiedad de la anterior, los que separados de la mezcla de la que proceden, son posteriormente vendidos a terceros, actividad que se corresponde con la definición de "explotador minero”, pues extrae sustancias minerales de carácter concesible como son las citadas, las 
que posteriormente enajena a terceros como la Empresa Nacional de Minería (Enami), transfiriendo de esa forma la propiedad de esos productos mineros, los que corresponden a cobre y molibdeno, sustancias minerales de carácter concesible, sin que tenga trascendencia alguna el estado productivo en que se encuentren.

DÉcimo: Que, como se aprecia, no se exige por el artículo 64 bis de la Ley de Impuesto a la Renta que se trate de sustancias respecto de las cuales se haya obtenido preliminarmente una concesión minera, toda vez que lo requerido por la norma es que sean de “carácter concesible”, esto es, susceptibles de ello, lo que excluye como exigencia su efectiva obtención.

UNDÉCIMO: Que lo señalando en las motivaciones precedentes permite comprobar la efectividad de los restantes errores de derecho esgrimidos por la defensa fiscal, en particular el referido a la vulneración del artículo 64 bis de la Ley de Impuesto a la Renta, pasando por alto sus exigencias y definiciones, prefiriendo -fuera de los casos permitidos-, normativa contenida en otras leyes, lo que llevó a los jueces del fondo a establecer erradamente la concurrencia de exigencias que no se encuentran en la disposición citada, desconociendo el tenor de la que debía aplicarse al caso propuesto.

Duodécimo: Que, los errores de derecho a que se ha hecho referencia en los considerandos anteriores han tenido además influencia sustancial en lo dispositivo del fallo impugnado, pues resulta de toda evidencia que, de no haberse incurrido en ellos, necesariamente los jueces de alzada debieron estimar que la sociedad reclamante de autos cumplía con los presupuestos legales para ser considerada sujeto pasivo del Impuesto Específico para la Actividad Minera.

Por estas consideraciones y de conformidad, además, con lo dispuesto en los artículos 764, 767 y 805 del Código de Procedimiento Civil, se acoge el recurso de casación en el fondo deducido en lo principal de fojas 241, por el abogado don Guillermo Neale Marín, en representación del Fisco de Chile, dirigido en contra de la sentencia de veinticinco de enero de dos mil once, que se lee a fojas 235 y siguientes, la que por consiguiente es nula y se la reemplaza por la que se dicta a continuación.

Regístrese.

Redacción a cargo del Ministro Sr. Juica.

Rol No 2.518-11.

Pronunciado por la Segunda Sala integrada por los Ministros Sres. Milton Juica A., Carlos Künsemüller L., Haroldo Brito C., Alfredo Pfeiffer R. y el abogado integrante Sr. Jorge Lagos G. No firman los Ministros Sres. Künsemüller y Brito, no obstante haber estado en la vista de la causa y acuerdo del fallo, por estar ambos en comisión de servicios.

Autorizada por la Ministro de Fe de esta Corte Suprema.

En Santiago, veintiocho de noviembre de dos mil doce, notifiqué en Secretaría por el Estado Diario la resolución precedente”. 
"Sentencia de Reemplazo.

Santiago, veintiocho de noviembre de dos mil doce.

De conformidad con lo dispuesto en el artículo 785 del Código de Procedimiento Civil, se dicta la siguiente sentencia de reemplazo.

\section{VISTOS:}

Se reproduce la sentencia en alzada.

Se replican de igual forma los considerandos sexto a décimo de la sentencia de casación que antecede.

Y atendido además lo dispuesto en los artículos 139 y 143 del Código Tributario, y los artículos 186 y siguientes del Código de Procedimiento Civil, se confirma la sentencia apelada de veinte de julio de dos mil diez, escrita a fojas 175 y siguientes.

\section{REGÍSTRESE Y DEVUÉLVASE.}

Redacción a cargo del Ministro Sr. Juica.

Rol No 2.518-11.

Pronunciado por la Segunda Sala integrada por los Ministros Sres. Milton Juica A., Carlos Künsemüller L., Haroldo Brito C., Alfredo Pfeiffer R. y el abogado integrante Sr. Jorge Lagos G. No firman los Ministros Sres. Künsemüller y Brito, no obstante haber estado en la vista de la causa y acuerdo del fallo, por estar ambos en comisión de servicios.

Autorizada por la Ministro de Fe de esta Corte Suprema.

En Santiago, veintiocho de noviembre de dos mil doce, notifiqué en Secretaría por el Estado Diario la resolución precedente.

\section{Comentario}

Este caso gira en torno a unos hechos (contratos de procesamiento de relaves entre concesionario minero y tercera empresa minera); unas partes determinadas (lo que nunca es irrelevante); un conjunto diferenciado de reglas que son llamadas a resolver el conflicto en cada Tribunal (Ley de Impuesto a la Renta, Código Tributario, Código de Minería, Código Civil) y, esencialmente, sobre el modo de interpretar la categoría de "explotador minero" (y, secundariamente, "venta"), contenida en el artículo 64 bis de la Ley de Impuesto a la Renta. Asimismo, se observa una determinada actuación del Servicio de Impuestos Internos (que liquida y gira impuestos específicos en contra de la tercera empresa); un camino administrativo (una consulta previa al Servicio y un Oficio); y un iter procesal con sentencias en contra, a favor y en contra del contribuyente reclamante. En cada fase, se observa una pesada artillería de argumentos de parte del contribuyente y del Servicio, y abigarrados razonamientos de cada Tribunal. 
Del análisis de contenido de las sentencias transcritas, intentando aislar con la mayor precisión los argumentos de las partes y de ambas Cortes, en este breve espacio es posible efectuar comentarios de dos extremos: sobre el caso; sobre las sentencias.

Las sentencias conducen este caso como si se tratara de una "caso fácil”, posible de resolverse sin otro medio que la subsunción (silogismo lógico) y atendiendo a definiciones (interpretación literal). En la sentencia de casación se observa que a una determinada interpretación que se efectúa de los hechos de la causa, es aplicada, sin más y parcialmente, el artículo 64 bis de la Ley de Impuesto a la Renta, considerando solo los elementos normativos que respaldan su tesis.

A mi parecer, los hechos de la causa son mucho más complejos que los sintetizados divergentemente en las sentencias de ambas Cortes. Los hechos seleccionados -aquellos elementos fácticos elegidos como relevantes-y su interpretación resultan esenciales para la decisión de los jueces. Las sentencias debieron abordar y discutir en detalle todos y no solo algunos hechos.

Por otro lado, los enfoques interpretativos de las normas, también divergentes en ambas Cortes, generan más consecuencias y son más complicados de lo que se expresa en las sentencias.

Ambas Cortes tratan este caso como un caso fácil. Pero se trata de un caso difícil. Un caso difícil requiere análisis y argumentaciones; y no se resuelve solo con silogismo lógico y definiciones e interpretaciones literales y afirmaciones autoritativas. Se puede observar, a partir de los caminos discursivos de ambas sentencias (los considerandos), que es posible decidir todo lo contrario en cada una de ellas, haciendo uso de los mismos argumentos. Esto pone de relieve que más de una solución válida era posible. Lo que exigía esta causa, y es a lo que renuncian las Cortes, es a exponer dialécticamente los argumentos que evalúen cada postura en términos de buscar la solución más plausible. Incluso era factible agregar otros argumentos y elementos y conclusiones dentro del discurso judicial.

La otra crítica, esta vez dirigida sobre la sentencia de casación, es que, sea cual sea la solución elegida por el Tribunal, debiera resguardarse la unidad y coherencia del sistema jurídico.

La Corte Suprema sustenta básicamente que se ha vulnerado por la Corte de Apelaciones el texto del artículo 64 bis de la Ley de Impuesto a la Renta, que no cabe acudir a norma suplementaria alguna (ex artículo $2^{\circ}$ del Código Tributario), por cuanto la Ley de la Renta "describe, precisa y define todos los elementos estructurales" del impuesto específico a la actividad minera, pues contiene normas especiales y diferentes en relación con la ley minera.

A mi juicio, la Ley de Impuesto a la Renta no establece un estatuto minero dentro de impuesto específico referido. Su regulación no llega a la autosuficiencia. La legislación tributaria, en este ámbito, se encuentra integrada a la regulación minera, y en particular al Código de Minería. La inteligibilidad de la imposición minera exige recurrir a este, pues este no establece normas arbitrarias, sino racionales y técnicas. Podemos discrepar de las normas mineras, pero esas son las leyes que se han consagrado en Chile y debiera fallarse en consideración a ellas. No existe un "estatuto especial minero" dentro del Derecho Tributario o 
de la Ley de Impuesto a la Renta. La racionalidad y unidad del sistema jurídico exige ubicar la imposición minera en los términos de las normativas mineras. De otro modo, se crearían verdaderos sistemas jurídicos paralelos.

Es probable que la Corte Suprema haya percibido un vacío normativo, un tax loophole, una grieta por la cual los contribuyentes pudieran eludir su deber de contribuir. En este tipo de casos, con elementos semejantes, ¿quién de los contratantes debe pagar el impuesto específico? ¿Puede ser que ambos se encuentren obligados; o ninguno de ellos?

La pregunta que cabe hacer es acerca de los mecanismos por los cuales los Tribunales (o el Servicio de Impuestos Internos) deben evitar que, mediante sutilezas o determinadas relaciones contractuales, se eluda el hecho imponible.

Lo interesante es que este caso muestra que la norma impositiva analizada está mal construida. Para los jueces, el impuesto específico a la actividad minera termina construyéndose sobre la base de una venta, más que sobre una estructura compleja que involucre los demás elementos de la obligación tributaria. Nótese que el sujeto pasivo de ella aparece discutido. 